\title{
CLOSURES OF DIRECT SUMS OF CLASSES OF OPERATORS
}

\author{
DON HADWIN
}

(Communicated by Palle E. T. Jorgensen)

Dedicated to Mary Ryan

\begin{abstract}
We prove that certain classes of Hilbert space operators that are direct sums of operators in specified classes are closed under sequential *-strong limits. One such example is the class of operators that are direct sums of operators that are either subnormal or have imaginary parts with spectrum contained in $[0,1] \cup[2,3]$.
\end{abstract}

Suppose $H$ is a Hilbert space, and let $B(H)$ denote the set of (bounded linear) operators on $H$. If $T \in B(H)$ and $M$ is a (closed linear) subspace of $H$, we say that $M$ reduces $T$ if $T(M) \subset M$ and $T\left(M^{\perp}\right) \subset M^{\perp}$. If $P$ is the orthogonal projection onto $M$, then $M$ reduces $T$ precisely when $T P=P T$. If $M$ reduces $T, A=T \mid M$, and $B=T \mid M^{\perp}$, we say that $T$ is the direct sum of $A$ and $B$, and we write $T=A \oplus B$. In this paper we consider classes of all operators that can be decomposed into direct sums of operators of specified types, and we show that, in a large number of cases, these classes are closed under sequential limits in various operator topologies. These results seem somewhat surprising, since the subspaces of decomposition are usually not continuous.

The operator topologies that we will consider are the norm, strong, *-strong, and weak operator topologies. A net $\left\{T_{n}\right\}$ of operators in $B(H)$ converges strongly to an operator $T$ if, for every vector $x$ in $H,\left\|T_{n} x-T x\right\| \rightarrow 0$. The net $\left\{T_{n}\right\}$ converges *-strongly to $T$ if both $T_{n} \rightarrow T$ and $T_{n}^{*} \rightarrow T^{*}$ strongly. The net $\left\{T_{n}\right\}$ converges weakly to $T$ if, for every $x, y$ in $H,\left(T_{n} x, y\right) \rightarrow$ $(T x, y)$.

For a sample result, suppose $\mathscr{S}$ is the class of all operators that can be decomposed into a direct sum of three operators (not all three summands must be present) $A \oplus B \oplus C$ such that $A$ is hyponormal $\left(A^{*} A-A A^{*} \geq 0\right), S^{-1} B^{*} S$ is subnormal (the restriction of a normal operator to an invariant subspace) with $\left\|S^{-1} B^{*} S\right\| \leq 12$ and $\|S\|\left\|S^{-1}\right\| \leq 37$, and $C^{2}$ is Hermitian. Note that the orthogonal decomposition $H=H_{A} \oplus H_{B} \oplus H_{C}$ is dependent on the operator $T$. Next note that such a decomposition is not unique (consider the identity operator). We show (Theorem 6 ) that $\mathscr{S}$ is closed under sequential *-strong

Received by the editors August 31, 1992.

1991 Mathematics Subject Classification. Primary 47B99; Secondary 47A20, 47A56. 
limits; it follows that $\mathscr{S}$ is closed under norm limits. However, $\mathscr{S}$ is not closed under strong sequential limits. In fact, every operator on an infinitedimensional space is the strong limit of a sequence of operators in $\mathscr{S}$ (since $\mathscr{S}$ contains all scalar multiples of the adjoints isometries).

A class $\mathscr{P}$ of operators is called a part class $[\mathrm{BFH}]$ precisely when it is closed under unitary equivalence and has the property that a direct sum of operators is in $\mathscr{P}$ if and only if each summand is in $\mathscr{P}$. It was shown in [BFH] that a class of operators $\mathscr{P}$ that is closed under unitary equivalence is a part class if and only if every Hilbert space operator can be uniquely decomposed into a direct sum $T=A \oplus B$ with $A$ in $\mathscr{P}$ and $B$ having no direct summands in $\mathscr{P}$.

The following result was proved in [H5, Theorem 5.1].

Proposition 1. Suppose $\mathscr{P}$ is a part class. The following are equivalent:

(1) $\mathscr{P}$ is closed under norm limits.

(2) $\mathscr{P}$ is closed under *-strong sequential limits.

(3) For every $T$ in $\mathscr{P}$ and every unital representation $\pi$ of $C^{*}(T)$, we have $\pi(T)$ is in $\mathscr{P}$.

A part class satisfying one of the above three properties is called a continuous part class. It was shown in [H1, Theorem 6.16] that if $r$ and $R$ are positive numbers, then the class $\mathscr{S}_{r, R}$ of operators $T$, for which there is an invertible operator $S$ with $S T S^{-1}$ subnormal, $\|S\|\left\|S^{-1}\right\| \leq r,\left\|S^{-1} T S\right\| \leq R$, is a continuous part class. Moreover, if we replace subnormal with normal in the definition of $\mathscr{S}_{r, R}$, we also obtain a continuous part class [H1, Theorem 6.11] that we will call $\mathscr{N}_{r, R}$.

We next consider part classes that are closed under strong sequential limits.

Proposition 2. For a continuous part class $\mathscr{P}$ the following are equivalent:

(4) $\mathscr{P}$ is closed under sequential strong limits.

(5) $\mathscr{P}$ is closed under restrictions to invariant subspaces.

(6) For every $T$ in $\mathscr{P}$ and every unital completely positive operator-valued map $\varphi$ on $C^{*}(T)$ such that $\varphi\left(T^{*} T\right)=\varphi\left(T^{*}\right) \varphi(T)$, we have $\varphi(T)$ is in $\mathscr{P}$.

Proof. (4) $\Rightarrow(5)$. Suppose $T \in B(H), T$ is in $\mathscr{P}, M$ is a subspace of $H$ such that $T(M) \subset M$, and $S=T \mid M$. Then $S \oplus S \oplus \cdots$ is the restriction of $T \oplus T \oplus \cdots$ to $M \oplus M \oplus \cdots$. Since $S$ is in $\mathscr{P}$ if and only if $S \oplus S \oplus \cdots$ is in $\mathscr{P}$, we can assume that $M$ is infinite dimensional. We can also assume that $H$ is separable, since $H$ is a direct sum of separable subspaces that reduce both $T$ and the projection onto $M$. We can therefore assume that both $H$ and $M$ are separable and infinite dimensional. It follows from [H4, Theorem 4.4] that $S=T \mid M$ is the strong limit of a sequence of operators that are unitarily equivalent to $T$. Hence $S$ is in $\mathscr{P}$ since $\mathscr{P}$ is a continuous part class.

$(5) \Rightarrow(6)$. Suppose $T$ is in $\mathscr{P}$ and $\varphi$ is a unital completely positive map from $C^{*}(T)$ into $B(M)$ for some Hilbert space $M$ such that $\varphi\left(T^{*} T\right)=$ $\varphi\left(T^{*}\right) \varphi(T)$. It follows from Stinespring's theorem [S] that there is a unital *-homomorphism $\pi: C^{*}(T) \rightarrow B(H)$, where $H$ is a Hilbert space containing $M$ such that, for every $A$ in $C^{*}(T), \varphi(A)=P \pi(A) \mid M$, where $P$ is the orthogonal projection onto $M$. It follows from $\varphi\left(T^{*} T\right)=\varphi\left(T^{*}\right) \varphi(T)$ that $M$ is an invariant subspace for $\pi(T)$; whence, $\varphi(T)$ is in $\mathscr{P}$, since it is the restriction of $\pi(T)$ to an invariant subspace. 
(6) $\Rightarrow(4)$. Suppose $\left\{T_{n}\right\}$ is a sequence in $\mathscr{P}$ and $T_{n} \rightarrow T$ strongly in $B(H)$. Let $\mathscr{H}=H \oplus H \oplus \cdots$. Since $T_{n} \rightarrow T$ strongly, it follows from the uniform boundedness theorem that $\sup _{n}\left\|T_{n}\right\|<\infty$. Let $T_{\infty}=T_{1} \oplus T_{2}$ $\oplus \cdots$ in $B(\mathscr{H})$. Define a sequence $\left\{V_{n}\right\}$ of isometrics from $H$ into $\mathscr{H}$ by $V_{1} f=f \oplus 0 \oplus 0 \oplus \cdots, V_{2} f=0 \oplus f \oplus 0 \oplus \cdots$, for each $f$ in $H$. Note that $V_{n}^{*} T_{\infty} V_{n}=T_{n}$ for $n=1,2, \ldots$. A standard compactness argument shows that there is a subsequence $\left\{V_{n_{k}}\right\}$ of $\left\{V_{n}\right\}$ such that, for every $A$ in $C^{*}\left(T_{\infty}\right)$, $\varphi(T)=$ (weak-operator) $\lim _{k} V_{n_{k}}^{*} T_{\infty} V_{n_{k}}$ exists. Then $\varphi$ is a unital completely positive map on $C^{*}\left(T_{\infty}\right)$ and $\varphi\left(T_{\infty}\right)=$ (weak-operator) $\lim _{k} T_{n_{k}}=T$. Since $T_{n_{k}} \rightarrow T$ strongly, it follows that $T_{n_{k}}^{*} T_{n_{k}} \rightarrow T^{*} T$ weakly. Hence $\varphi\left(T_{\infty}^{*} T_{\infty}\right)=$ $\varphi\left(T_{\infty}^{*}\right) \varphi\left(T_{\infty}\right)$. It follows from (6) that $T$ is in $\mathscr{P}$.

A part class satisfying one of the conditions (4)-(6) is called a strongly continuous part class. The classes of subnormal operators and hyponormal operators and the class $\mathscr{S}_{r, R}$ defined above are strongly continuous part classes.

The final part classes we consider are the ones that are closed under weak limits. If $T \in B(H), M$ is a subspace of $H$, and $P$ is the orthogonal projection onto $M$, then the compression of $T$ to $M$ is the operator $P T \mid M$ in $B(M)$. The following proposition is proved in a manner that is very similar to that of the preceding proposition, and it is therefore omitted.

Proposition 3. For a continuous part class $\mathscr{P}$ the following are equivalent:

(7) $\mathscr{P}$ is closed under weak sequential limits.

(8) $\mathscr{P}$ is closed under compressions.

(9) For every $T$ in $\mathscr{P}$ and every unital completely positive operator-valued map $\varphi$ on $C^{*}(T)$, we have $\varphi(T)$ is in $\mathscr{P}$.

Continuous part classes behave with direct integrals like part properties behave with direct sums. Suppose $H$ is a separable Hilbert space and $(\Omega, \mu)$ is a complete finite measure space. We define $L^{2}(\mu, H)$ to be the Hilbert space of all measurable functions $f$ from $\Omega$ to $H$ such that $\|f\|^{2}=\int_{\Omega}\|f(\omega)\|^{2} d \mu(\omega)$ $<\infty$. We let $L^{\infty}(\mu, B(H))$ denote the set of all strongly measurable functions $\varphi: \Omega \rightarrow B(H)$ such that $\|\varphi\|_{\infty}=$ ess-sup $\|\varphi(\omega)\|<\infty$. Each $\varphi$ in $L^{\infty}(\mu, B(H))$ induces an operator $T$ on $L^{2}(\mu, H)$ defined by $T(\varphi)(\omega)=$ $\varphi(\omega) f(\omega)$. The operator $T$ is called the direct integral of the operators $T_{\omega}=$ $\varphi(\omega)$, and we write $T=\int^{\oplus} T_{\omega} d \mu(\omega)$.

A result from [H2] (see [H3, Theorem 6.2]) relates direct integrals and direct sums in terms of approximate equivalence. Two operators $A$ and $B$ are approximately equivalent [V], denoted $A \sim_{a} B$, if $B$ is a norm limit of operators that are unitarily equivalent to $A$. The results in [H2, H3] state that if $T=\int^{\oplus} T_{\omega} d \mu(\omega)$, then

(i) either $T_{\omega}$ is a summand of $T$ or $T \sim_{a} T \oplus T_{\omega}$ a.e. $(\mu)$, and

(ii) $T \sim_{a} T_{\omega_{1}} \oplus T_{\omega_{2}} \oplus \cdots$ for some sequence $\left\{\omega_{n}\right\}$ in $\Omega$.

The following proposition is an immediate consequence of these ideas, since a continuous part class is closed under approximate equivalence.

Proposition 4. Suppose $\mathscr{P}$ is a continuous part class. $A$ direct integral $\int^{\oplus} T_{\omega} d \mu(\omega)$ on a separable Hilbert space is in $\mathscr{P}$ if and only if almost every $T_{\omega}$ is in $\mathscr{P}$. 
Another key ingredient in our results is the following simple result concerning irreducible representations. In [E] Ernest defined a spectrum for an operator $T$, which we call the Ernest spectrum and is defined to be the set of unitary equivalence classes of the operators $\pi(T)$ with $\pi$ an irreducible representation of $C^{*}(T)$. The following lemma says that the Ernest spectrum of a finite direct sum of operators is the union of the Ernest spectra of the summands. Recall [H5] that $\psi$ is a continuous decomposable function if there is a sequence $\left\{p_{n}(x, y)\right\}$ of noncommutative polynomials such that $\left\|\psi(T)-p_{n}\left(T, T^{*}\right)\right\| \rightarrow 0$ uniformly on bounded sets on $B(H)$, where $H$ is any Hilbert space.

Lemma 5. Suppose $T=T_{1} \oplus T_{2} \oplus \cdots \oplus T_{n}$ and $\pi: C^{*}(T) \rightarrow B\left(H_{\pi}\right)$ is an irreducible representation. Then there is a $k, 1 \leq k \leq n$, and an irreducible representation $\rho$ of $C^{*}\left(T_{k}\right)$ such that $\rho\left(T_{k}\right)=\pi(T)$.

Proof. It suffices to prove the lemma when $n=2$. The rest follows by induction. Assume via contradiction that no such $k, 1 \leq k \leq 2$, and $\rho$ exist. It follows from [H5, Corollary 3.2] that there are nonnegative continuous decomposable functions $\psi_{1}, \psi_{2}$ such that $\psi_{1}\left(T_{1}\right)=0, \psi_{2}\left(T_{2}\right)=0$ and $\psi_{1}(\pi(T)) \neq 0, \psi_{2}(\pi(T)) \neq 0$. Hence $\psi_{1}(T) C^{*}(T) \psi_{2}(T)=\{0\}$, which, by applying $\pi$, yields $\psi_{1}(\pi(T)) C^{*}(\pi(T)) \psi_{2}(\pi(T))=\{0\}$. Since $\pi$ is irreducible, $C^{*}(\pi(T))$ is strongly dense in $B\left(H_{\pi}\right)$. This contradicts the fact that $\psi_{1}(\pi(T)) \neq 0$ and $\psi_{2}(\pi(T)) \neq 0$.

If $\mathscr{P}_{1}, \mathscr{P}_{2}, \ldots, \mathscr{P}_{n}$ is a finite collection of part classes, we define their direct sum $\mathscr{P}_{1} \oplus \mathscr{P}_{2} \oplus \cdots \oplus \mathscr{P}_{n}$ to be the class of operators that can be decomposed into a direct sum of operators in $\mathscr{P}_{1} \cup \cdots \cup \mathscr{P}_{n}$. It is easily seen that a finite direct sum of part classes is a part class. Our main result concerns direct sums of continuous part classes.

Theorem 6. Suppose the part class $\mathscr{P}$ is the direct sum of the part classes $\mathscr{P}_{1}, \mathscr{P}_{2}, \ldots, \mathscr{P}_{n}$. If each $\mathscr{P}_{k}$ is a continuous part class, then $\mathscr{P}$ is a continuous part class.

Proof. Suppose $T=T_{1} \oplus T_{2} \oplus \cdots \oplus T_{n}$, with $T_{k}$ in $\mathscr{P}_{k}$ for $1 \leq k \leq n$, and suppose that $\pi: C^{*}(T) \rightarrow B\left(H_{\pi}\right)$ is a representation. Since $\mathscr{P}$ is a part class and $\pi$ is a direct sum of representations on separable Hilbert spaces, we can assume that $H_{\pi}$ is separable. However, every representation on a separable Hilbert space is a direct sum of direct integrals of irreducible representations [D], so we can assume that $\pi$ is a direct integral of irreducible representations.

First suppose $\pi$ is irreducible. It follows from Lemma 5 that there is a $k, 1 \leq k \leq n$, and a representation $\rho$ on $C^{*}\left(T_{k}\right)$ such that $\rho\left(T_{k}\right)=\pi(T)$; whence, $\pi(T)$ is in $\mathscr{P}_{k}$.

For the case in which $\pi$ is a direct integral of irreducible representations, we obtain $\pi: C^{*}(T) \rightarrow B\left(L^{2}(\mu, H)\right), \pi(A)=\int^{\oplus} \pi_{\omega}(A) d \mu(\omega)$ for every $A$ in $C^{*}(T)$, and each $\pi_{\omega}$ is an irreducible representation. Since each $\mathscr{P}_{k}$ is closed under *-strong sequential limits, we know $\mathscr{P}_{k} \cap B(H)$ is a Borel set for $1 \leq k \leq n$. Hence $\left\{\omega \in \Omega: T_{\omega}\right.$ is in $\left.\mathscr{P}_{k}\right\}$ is a measurable subset of $\Omega$ for $1 \leq k \leq n$. Thus, by Proposition $4, \pi(T)$ is in $\mathscr{P}$.

It seems at first glance that the proof of Theorem 6 might be extendable to prove the analogues for strongly continuous and weakly continuous part classes. However, neither of these analogues is true, as the following examples show. 
Example. Let $\mathscr{P}_{1}$ be the class of all isometries, let $\mathscr{P}_{2}$ be the class of all operators with norm at most $1 / 2$, and let $\mathscr{P}_{3}$ denote the class of all Hermitian operators. Then, since $\mathscr{P}_{1}$ is closed under restrictions to invariant subspaces and $\mathscr{P}_{2}$ and $\mathscr{P}_{3}$ are closed under compressions, $\mathscr{P}_{1}$ is strongly continuous and both $\mathscr{P}_{2}$ and $\mathscr{P}_{3}$ are weakly continuous. However, $\mathscr{P}_{1} \oplus \mathscr{P}_{2}$ is not strongly continuous, and $\mathscr{P}_{2} \oplus \mathscr{P}_{3}$ is not weakly continuous.

To see this, let $S$ be the unilateral shift operator, defined on an orthonormal basis $\left\{e_{1}, e_{2}, \ldots\right\}$ by $S e_{n}=e_{n+1}$. Then $T=S \oplus \frac{1}{2} S$ is in $\mathscr{P}_{1} \oplus \mathscr{P}_{2}$. Let $M$ be the closed linear span of $\left\{e_{n} \oplus e_{n} / 2^{n}: n \geq 1\right\}$. Then $T(M) \subset M$, and $T \mid M$ is a weighted unilateral weighted shift that is irreducible but is neither an isometry nor an operator with norm at most $\frac{1}{2}$. Hence $T \mid M$ is not in $\mathscr{P}_{1} \oplus \mathscr{P}_{2}$. Thus $\mathscr{P}_{1} \oplus \mathscr{P}_{2}$ is not strongly continuous.

Let $A$ be a normal operator whose spectrum is the closed disk centered at 0 with radius $\frac{1}{2}$, and let $B=2$. Then $A \oplus B$ is in $\mathscr{P}_{2} \oplus \mathscr{P}_{3}$, but the numerical range of $A \oplus B$, being dense in the closed convex hull of its spectrum, contains a complex number $\lambda$ that has absolute value greater than $\frac{1}{2}$ and is not real. However, $\lambda$ is a compression of $A \oplus B$ that is not in $\mathscr{P}_{2} \oplus \mathscr{P}_{3}$. Thus $\mathscr{P}_{2} \oplus \mathscr{P}_{3}$ is not weakly continuous.

The author expresses his gratitude to the National Science Foundation for its support while this research was undertaken.

\section{REFERENCES}

[BFH] A. Brown, C.-K. Fong, and D. W. Hadwin, Parts of operators on Hilbert space, Illinois J. Math. 22 (1978), 306-314.

[D] J. Dixmier, $C^{*}$-algebras, North-Holland, Amsterdam, 1977.

[E] J. Ernest, Charting the operator terrain, Mem. Amer. Math. Soc., no. 171, Amer. Math. Soc., Providence, RI, 1976.

[H1] D. W. Hadwin, An asymptotic double commutant theorem for $C^{*}$-algebras, Trans. Amer. Math. Soc. 244 (1978), 273-297.

[H2] _ Approximating direct integrals of operators by direct sums, Michigan Math. J. 25 (1978), 123-127.

[H3] - Non-separable approximate equivalence, Trans. Amer. Math. Soc. 266 (1981), 203-231.

[H4] , Completely positive maps and approximate equivalence, Indiana Univ. Math. J. 36 (1987), 211-228.

[H5] _ Continuous functions of operators: a functional calculus, Indiana Univ. Math. J. 27 (1978), 113-125.

[S] W. Stinespring, Positive functions on $C^{*}$-algebras, Proc. Amer. Math. Soc. 6 (1955), 211-216.

[V] D. Voiculescu, A non-commutative Weyl-von Neumann theorem, Rev. Roumaine Math. Pures Appl. 21 (1976), 97-113.

Department of Mathematics, University of New Hampshire, Durham, Hew Hampshire 03824

E-mail address: dwhadwin@christa.unh.edu 